\title{
Arithmetical Fourier transforms and Hilbert space: Restoration of the lost legacy
}

\author{
J.-W. Feng, S. Kanemitsu and T. Kuzumaki
}

\begin{abstract}
In this survey-type paper we show that the seemingly unrelated two fields - Chebyshev-Markov expansion (CME) [On83] and Arithmetical Fourier Transform (AFT) [Che10] - are indeed different looks of one entity, by the plausible missing link-Romanoff-Wintner theory (RWT). RWT generalizes both approaches, CME and AFR, and was developed in [Wi44] and [Ro51a], [Ro51b] which were written independently. These two lost researches are very closely related and effective for producing new number-theoretic identities. Cf. [CKT09] for fragmental restoration of them.
\end{abstract}

Keywords. Chebyshev-Markov expansion (CME), Arithmetical Fourier Transform (AFT), Hilbert space, Möbius inversion. 2010 Mathematics Subject Classification. 42A16, 11A25, 01A55.

\section{Introduction}

Two generations are long enough for the mathematical community to forget what was found 50 years ago, which we call every 50 years phenomenon. One of the most famous examples is the case of the Lerch-Chowla-Selberg formula for a relation between special values of the gamma- and thetafunctions (which arises by comparing the Kronecker limit formula and decomposition theorem). The case of the Dedekind eta-transformation formula (ETF) and discriminant-functions transformation formula (DTF) is well restored. Ramanujan would have proved the ETF [KaTs14, pp. 80-95]. In 1968 Chowla proved the DTF and in 1970 Weil proved the ETF by the functional equation for the relevant zeta-function. The proof of the DTF depending on Eisenstein series is given in the most recent inspirational book of Chan [Ch20, §6.5, pp.71-77]. With Ramanujan there are associated many sources for further research, and so there exist proper restoration of his legacy. Hardy-Ramanujan J. is one of the welcome trends of revival of Ramanujan's ideas and thoughts.

At this occasion, we restore another legacy which is not well-known but is to be better known. It is the case of Chebyshev-Markov Expansion (CME) and Romanoff-Wintner Theory (RWT). We have restored RWT very fragmentarily in [CKLW09]. One year later, Chen's book [Che10] appeared in Tsinghua Univ. Phys. Ser. and only recently it came to our attention in view of part of its title Möbius inversion. Chen's main purpose [Che10] is the revival of Arithmetical Fourier Transform (AFT) or the Bruns' algorithm [Br1903] in the setting of computer algorithms, cf. toward the end of $\S 4 .$. AFT is analogous to the CME. True that the author [Che10] has rather diligently searched for lost references after 1983 (the year [On83] was published), especially [Chb1851] and [Wi47]. The former is not mentioned in [On83] but is the very genesis of CME (cf. the passage after Table 1 below), while the latter is not easily available since it is a private edition. But [Che10] misses [On83] etc. and does not mention CME nor its generalization RWT, [Ro51a], [Ro51b], [Wi44], all the more [CKLW09], i.e. the right half of Figure 1 is missing, so that if not recorded properly, only AFT will survive since books are long-lived, papers being very short-lived. Remark that Chebyshev $\sim 1850$, Markov, Bruns $\sim 1900$, Romanoff, Wintner $\sim 1950$, Chakraborty et al, Chen $\sim 2010$, intervals being nearly 50 years.

Thus our aim is to restore the invaluable knowledge from the past and streamline their relation in a proper perspective and framework in which the Möbius inversion plays a central role. In RomanoffWintner theory of an orthonormal system (ONS) in Hilbert space, the Möbius inversion works as an

We thank episciences.org for providing open access hosting of the electronic journal Hardy-Ramanujan Journal 
alternative for the classical Gram-Schmidt orthogonalization method if the sequence has the $D_{g^{-}}$ property, cf. §3., [Ro51a, p.6], [KaKi95, p.17].

We give mostly survey type presentations save for $\S 3$.A. where we state some examples of an ONS in a Hilbert space á la Romanoff. In §2. we state description of CME, in §3., Romanoff-Wintner theory of ONS in a Hilbert space, in $\S 4$., the Discrete Fourier Transform (DFT) and brief description of AFT and the Fast Fourier Transform (FFT). The following Figure and Table will make it easier to go through the paper.

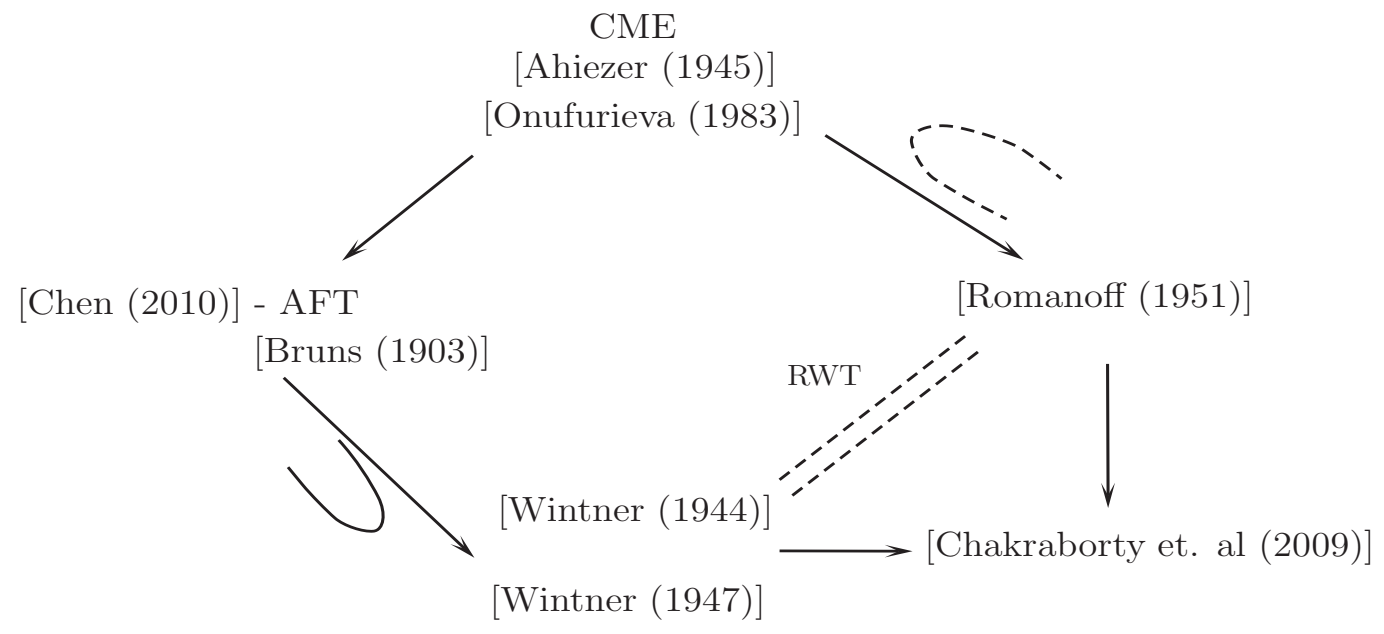

Figure 1. Relation among literature

\begin{tabular}{|l|c|c|}
\hline short-hand & full name & related subject matter \\
\hline AFT & Arithmetical Fourier Transform & Bruns' algorithm, CME, FFT \\
\hline CME & Chebyshev-Markov Expansion & RWT \\
\hline DFT & Discrete Fourier Transform & for fast computation, AFT \& FFT \\
\hline FFT & Fast Fourier Transform & Cooley-Tukey algorithm, AFT \\
\hline RWT & Romanoff-Wintner Theory & [CKLW09] \\
\hline
\end{tabular}

Table 1. List of abbreviations

In relation to Remark 3.10 below, we note the following in anticipation of further study. As mentioned above, Chen [Che10] refers to a very classical paper of Chebyshev [Chb1851, pp.96-99] which is not referred to in [On83]. It deals with the Fourier expansion of the triangular waves and their Möbius inversion. This must be the genesis of CME and shows how [Che10] is thorough.

All similar waves can be expressed as a linear combination of the first periodic Bernoulli polynomial, or the imaginary part of the boundary function $\ell_{1}(x)$ of the polylogarithm function of order 1 (after analytic continuation, cf. below). Cf. [KaTs07, pp.136-150]. In general, the polylogarithm function $\operatorname{Li}_{s}(z)=\sum_{n=1}^{\infty} \frac{z^{n}}{n^{s}}$ is defined inside the unit circle $|z|<1$. It is probably Wintner [Wi41] who studied the boundary function of Lambert series, which naturally led to the boundary function of $\operatorname{Li}_{s}(z)$, i.e. the polylogarithm function of the complex exponential argument $\ell_{s}(x)=\sum_{n=1}^{\infty} \frac{e^{2 \pi i n x}}{n^{s}}$. Cf. e.g. [Lew80]. It is another name for the Lerch zeta-function, cf. [LaGa02]. As has been wellestablished cf. e. g. [WAK20], the Lerch zeta-function being a Fourier series as well as a zeta-function that satisfies the functional equation, turns out to be quite useful.

Short character sums have been the object of enormous amount of studies since the result of Dirichlet for the $\frac{1}{4}$-sum. But this does not fall in every 50 years category since there were so many rediscoveries and even Dirichlet's result is re-discovered. It is Yamamoto's decisive work [Ya77], after an 
intermediate summit of [Be76], that enables us to express any short character sums with polynomial or Clausen function weight in terms of linear combination of $L(1, \chi)$. This has not properly been cited in literature but is decisive. Only recently, the underlying structure of Yamamoto's results has been revealed as a consequence of the boundary Fourier series, the Lerch zeta-function by [WMK20]. In Remark 3.10, we will see an example of the correspondence between $\operatorname{Li}_{1}(z)$ and its boundary function $\ell_{1}(x)$.

\section{Chebyshev-Markov expansion}

Onuferieva [On83, esp. p.268 ] is the most informative reference on the Chebyshev-Markov expansion (CME) up to 1983 while [Che10, esp. p.126] contains information on modern applications of Arithmetical Fourier Transform (AFT) to signal processing, VLSI etc. Both refer to Bruns [Br1903] but Liebmann [Li1909] and Müller [M54] are referred to only in [On83] in which the author criticizes Romanoff [Ro51a], [Ro51b] and his students for not referring to the fundamental work of Chebyshev [Chb1859] ([Chb1858] is a short communication) and Markov [Ma1898]. As is mentioned on [On83, p.273], Ahiezer [Ah45] and [Ah47, p.511], had been the only sources about Chebyshev-Markov systems. Indeed, Ahiezer [Ah45, pp.38-40] is as informative as Onufrieva [On83] and in some sense more authoritative, referring to the famous Rademacher system [Rad1922], which is a part of the Chebyshev-Markov system.

Wintner [Wi47] is a reformulation of Bruns' algorithm (which is developed independently of [Br1903]).

Onufrieva mistakingly refers to the paper [Ma1895] which is not the one in question and the paper should be [Ma1898]. It seems that the author missed the second line in the title.

These two fields - CME and AFT — look almost irrelevant, the missing link being probably [Wi44] which is written independently of [Ro51a, Ro51b]. Wintner's theory [Wi44] is not mentioned in [On83] nor in [Che10] but is closely related to Romanoff's theory and we summed them up to write a paper [CKLW09].

As is mentioned [On83], toward the end of his paper [Ma1898], Markov established a trigonometric analogue of Chebyshev's method.

We state the outline of CME.

Let $\sigma(x)$ be a non-decreasing function on $I=[0,1]$, say. Then the system of functions $\left\{r_{n}(x)\right\}$ and $\left\{s_{n}(x)\right\}(n=0,1, \cdots)$ is called biorthogonal if

$$
\int_{I} r_{i}(x) s_{j}(x) \mathrm{d} \sigma(x)=\delta_{i j}
$$

We write $\left\{r_{n} ; s_{n}\right\}$ to indicate biorthogonality. For any function $f(x)$ for which the integrals below exist, we have a formal expansion

$$
f(x) \sim \sum_{n=0}^{\infty} A_{n} r_{n}(x), \quad A_{n}=\int_{I} f(x) s_{n}(x) \mathrm{d} \sigma(x) .
$$

In what follows we choose $\sigma(x)=x$ and we let $\mu$ denote the Möbius function. Following the notation of [On83], we put for $n \in \mathbb{N}$

$$
\begin{aligned}
& \psi_{0}(x)=1, \quad \psi_{n}(x)=\frac{\pi}{2} \sum_{d \mid n} \frac{\mu(d) \chi(d)}{d} \cos \frac{2 \pi n x}{d}, \\
& \varphi_{n}(x)=\frac{\pi}{2} \sum_{d \mid n} \frac{\mu(d) \chi^{2}(d)}{d} \sin \frac{2 \pi n x}{d}
\end{aligned}
$$


where $\chi=\chi_{4}$ is the primitive Dirichlet character mod 4 and

$$
u_{0}(x)=1, \quad u_{n}(x)=\operatorname{sign} \cos 2 \pi n x, \quad v_{n}(x)=\operatorname{sign} \sin 2 \pi n x,
$$

where $\operatorname{sign} y=\frac{y}{|y|}$ or 0 according as $y \neq 0$ or $y=0$. Markov proved that the system $\left\{\psi_{0}, \psi_{n}, \varphi_{n} ; u_{0}, u_{n}, v_{n}\right\}=$ $\left\{\psi_{0}, \psi_{1}, \varphi_{1}, \psi_{2}, \varphi_{2}, \cdots ; u_{0}, u_{1}, v_{1}, u_{2}, v_{2}, \cdots\right\}$ is biorthogonal on $[0,1]$. By some change of variable, $\left\{\varphi_{n} ; v_{n}\right\}$ coincides with the system constructed by Chebyshev and so the system is called the ChebyshevMarkov (biorthogonal) system [Ah45, p.39]. Suppose $f \in L^{2}(0,1)$. Then (2.2) reads

$$
f(x) \sim A_{0}+\sum_{n=1}^{\infty}\left(A_{n} \psi_{n}(x)+B_{n} \varphi_{n}(x)\right)
$$

where

$$
A_{0}=\int_{I} f(x) \mathrm{d} x, \quad A_{n}=\int_{I} f(x) \operatorname{sign} \cos 2 \pi n x \mathrm{~d} x, \quad B_{n}=\int_{I} f(x) \operatorname{sign} \sin 2 \pi n x \mathrm{~d} x .
$$

Onufrieva calls this the Chebyshev-Markov expansion (CME). If $a_{m}, b_{m}$ are Fourier coefficients of $f(x)$, then

$$
A_{n}=\frac{2}{\pi} \sum_{k=1}^{\infty} \frac{\chi(k)}{k} a_{k n}, \quad B_{n}=\frac{2}{\pi} \sum_{k=1}^{\infty} \frac{\chi^{2}(k)}{k} b_{k n}
$$

as proved by Liebmann [Li1909]. By Möbius inversion, (2.7) is inverted into

$$
a_{n}=\frac{\pi}{2} \sum_{k=1}^{\infty} \frac{\mu(k) \chi(k)}{k} A_{k n}, \quad b_{n}=\frac{\pi}{2} \sum_{k=1}^{\infty} \frac{\mu(k) \chi^{2}(k)}{k} B_{k n}
$$

formally by Bruns [Br1903]. Rigorously, this can be proved by the method of Ahiezer [Ah47] by which he proved completeness of the system $\{\operatorname{sign} \sin 2 \pi n x\}$ on $\left[0, \frac{1}{2}\right]$. A more cumbersome proof is given by Müller [M54]. Another proof is given in [On83, pp.271-273] which depends on the integrated form of (2.5) developed in [KiOn61].

We restate the proof. Let $(x \in[0,1])$

$$
F(x)=\int_{0}^{x}\left(f(t)-A_{0}\right) \mathrm{d} t .
$$

Then

$$
F(x)=A_{0}^{*}+\sum_{n=1}^{\infty}\left(A_{n}^{*} g_{n}(x)+B_{n}^{*} h_{n}(x)\right)
$$

where the series is uniformly convergent on $I$ and $A_{n}^{*}=-\frac{B_{n}}{4 n}, B_{n}^{*}=\frac{A_{n}}{4 n}$ and where

$$
\begin{aligned}
& g_{n}(x)=-4 n \int_{0}^{x} \varphi_{n}(t) \mathrm{d}=\sum_{d \mid n} \mu(d) \chi^{2}(d) \cos \frac{2 \pi n x}{d}+\left\{\begin{array}{cc}
-1 & n=2^{\nu} \\
0 & n \neq 2^{\nu}
\end{array}\right. \\
& h_{n}(x)=4 n \int_{0}^{x} \psi_{n}(t) \mathrm{d} t=\sum_{d \mid n} \mu(d) \chi(d) \sin \frac{2 \pi n x}{d} .
\end{aligned}
$$

Note that

$$
\int_{0}^{1} F(t) \cos 2 \pi m t \mathrm{~d} t=-\frac{1}{2 \pi m} \int_{0}^{1} f(t) \sin 2 \pi m t \mathrm{~d} t=-\frac{b_{m}}{4 \pi m}
$$

and

$$
\int_{0}^{1} F(t) \sin 2 \pi m t \mathrm{~d} t=\frac{1}{2 \pi m} \int_{0}^{1} f(t) \cos 2 \pi m t \mathrm{~d} t=\frac{a_{m}}{4 \pi m} .
$$


It follows that the Fourier coefficients of $F(x)$ are constant multiples of those of $f(x)$. Hence we may simply find the Fourier coefficients of the right-hand side of (2.9). This method (more generally summability method) may work in many other problems. E.g. for arithmetical Fourier series [KaTs14, pp.196-206], integrated ones follow from the functional equation but in order to assure uniform convergence of differentiated series, estimates for exponential sums are needed which are as deep as those for the prime number theorem.

In the same paper [KiOn61], they proved that if $f$ is either of $\operatorname{Lip}^{\alpha}, \alpha>0$ and of bounded variation or $\operatorname{Lip}^{\alpha}, \alpha>\frac{1}{2}$, then the series (2.5) is absolutely and uniformly convergent to $f(x)$ while the integrated form holds with equality as long as $f \in L^{2}(0,1)$, the Fourier coefficients having the reducing factor $\frac{1}{n}$.

\section{Romanoff-Wintner theory of ONS in a Hilbert space}

The purpose of the present section is to show that some number-theoretic identities have very natural hidden structure, i.e. the Parseval identity, that is why they are to hold. To uncover such phenomena, we are to discover suitable complete orthonormal systems. We state essentials of RWT to find complete ONS. Note that in the case of finite-dimensional normed vector spaces, all orthonormal systems (ONS) consisting of the dimension-number of elements are complete and therefore, we immediately obtain the Parseval identities. On the other hand, in the Hilbert space $L^{2}(0,1)$, the space of all squareintegrable functions, completeness is essential to attain the Parseval identity. We follow [Ro51a] (and [KaKi95], which is an annotated translation thereof), [Ro51b] and [Wi44].

Definition 1. A sequence $\left\{f_{n}\right\}$ of a Hilbert space $H$ is said to have the $D_{g}$ property if

$$
\left(f_{m}, f_{n}\right)=g((m, n)),
$$

where $(m, n)$ means the $g c d$ of $m$ and $n$ and $g$ is a given function of positive integer argument.

Throughout in what follows, we mean by $H$ a (complex) Hilbert space.

Theorem 3.1. ([Ro51a, Theorem 3]) Suppose $\omega(n) \neq 0$ is a totally multiplicative complex-valued function with finite square norm

$$
w:=\sum_{n=1}^{\infty}|\omega(n)|^{2}<\infty
$$

and that $\left\{\alpha_{n}\right\}$ is an ONS in H. Let

$$
f_{n}:=\overline{\omega(n)}^{-1} \sum_{k=1}^{\infty} \omega(k) \alpha_{k n} .
$$

Then $\left\{f_{n}\right\}$ has the $D_{g}$ property, where

$$
g(n)=\frac{w}{|\omega(n)|^{2}}
$$

Theorem 3.2. ([Ro51a, Theorem 2]) Suppose two sequences $\left\{f_{n}\right\}$ and $\left\{\psi_{n}\right\}$ of a Hilbert space are connected by

$$
\psi_{n}=\frac{1}{\sqrt{G(n)}} \sum_{d \mid n} \mu\left(\frac{n}{d}\right) f_{d},
$$

where $G(n)>0$. Then $\left\{\psi_{n}\right\}$ forms an ONS if and only if $\left\{f_{n}\right\}$ has the $D_{g}$-property, where

$$
G(n)=\sum_{d \mid n} \mu\left(\frac{n}{d}\right) g(d) .
$$


Combining Theorem 3.1 and Theorem 3.2 with $G(n)=w \Omega(n)$ in (3.19), we deduce the following theorem, where $\Omega(n)$ is given in (3.21):

Theorem 3.3. ([Ro51a, Theorem 4]) Suppose $\alpha_{n}$ is an ONS in $H$ and that $\omega(n) \neq 0$ is as in Theorem 3.1. Let

$$
\psi_{n}:=(w \Omega(n))^{-\frac{1}{2}} \sum_{d \mid n} \mu\left(\frac{n}{d}\right) f_{d}=(w \Omega(n))^{-\frac{1}{2}} \sum_{d \mid n} \mu\left(\frac{n}{d}\right) \overline{\omega(d)^{-1}} \sum_{k=1}^{\infty} \omega(k) \alpha_{k d},
$$

where

$$
\Omega(n)=\sum_{d \mid n} \mu\left(\frac{n}{d}\right)|\omega(d)|^{-2}=|\omega(n)|^{-2} \prod_{p \mid n}\left(1-|\omega(p)|^{2}\right) .
$$

Then $\left\{\psi_{n}\right\}$ forms an ONS.

Theorem 3.4. ([Ro51a, Theorem 5]) Notation being the same as in Theorem 3.3, suppose that $\left\{\alpha_{n}\right\}$ is a complete ONS in the subspace $H^{\prime}$ of $H$. Then $\left\{\psi_{n}\right\}$ in (3.20) forms a complete ONS in $H^{\prime}$.

\section{A. Example of a Hilbert space}

Lemma 3.5. If $f(z)$ is analytic in an open disc $D_{r}:|w-z|<r$, then

$$
|f(z)|^{2} \leq \frac{1}{\pi r_{0}^{2}} \iint_{\overline{D_{r_{0}}}}|f(w)|^{2} \mathrm{~d} x \mathrm{~d} y, \quad w-z=x+i y
$$

for $0<r_{0}<r$, with the bar denoting the closure.

Proof. Let

$$
f(w)=\sum_{n=0}^{\infty} a_{n}(w-z)^{n} \quad w \in D_{r}
$$

be the Taylor expansion. Then

$$
f(z)=a_{0} .
$$

Writing $x+i y=w-z=\rho e^{i \theta}$ and multiplying $f(w)$ by $\overline{f(w)}$, we obtain

$$
f(w) \overline{f(w)}=\sum_{m, n=0}^{\infty} a_{m} \overline{a_{n}} \rho^{m+n} e^{(m-n) i \theta}
$$

which is absolutely and uniformly convergent in $0 \leq \rho \leq r_{0}<r, 0 \leq \theta \leq 2 \pi$. Hence we may integrate (3.24) termwise to obtain

$$
\begin{aligned}
& \iint_{\overline{D_{r_{0}}}}|f(w)|^{2} \mathrm{~d} x \mathrm{~d} y=\int_{0}^{r_{0}} \int_{0}^{2 \pi}|f(w)|^{2} \mathrm{~d} \theta \rho \mathrm{d} \rho=2 \pi \sum_{n=0}^{\infty}\left|a_{n}\right|^{2} \int_{0}^{r_{0}} \rho^{2 n+1} \mathrm{~d} \rho \\
& =2 \pi \sum_{n=0}^{\infty}\left|a_{n}\right|^{2} \frac{1}{2 n+2} r_{0}^{2 n+2} .
\end{aligned}
$$

Hence the last term $\geq \pi r_{0}^{2}\left|a_{0}\right|^{2}=\pi r_{0}^{2}|f(z)|^{2}$ and the lemma follows.

Theorem 3.6. Let $A^{2}(D)$ denote the space of all functions $f(z)$ which are (one-valued) and analytic on a (bounded) domain $D$ satisfying

$$
\iint_{D}|f(z)|^{2} \mathrm{~d} x \mathrm{~d} y<\infty, \quad z=x+i y
$$


Define operations as usual, i.e. $(f+g)(z)=f(z)+g(z)$ and $(a f)(z)=a f(z)$ and introduce the inner product

$$
(f, g)=\iint_{D} f(z) \overline{g(z)} \mathrm{d} x \mathrm{~d} y .
$$

Then $A^{2}=A^{2}(D)$ forms a Hilbert space, i.e. every Cauchy sequence is convergent.

Proof. That $f+g \in A^{2}$ follows from the inequality $|\alpha+\beta|^{2} \leq 2\left(|\alpha|^{2}+|\beta|^{2}\right)$. That $(f, g)$ in (3.27) is finite follows from the inequality $|\alpha \beta| \leq \frac{1}{2}\left(|\alpha|^{2}+|\beta|^{2}\right)$ and one can easily check this is an inner product, defining the norm \|\| . It remains to prove completeness.

Suppose $\left\{f_{n}\right\} \subset A_{2}$ is a Cauchy sequence, i.e.

$$
\lim _{m, n \rightarrow \infty}\left\|f_{n}-f_{m}\right\|=0 .
$$

We may choose $\nu_{1}$ so that for $n>\nu_{1}$ we have

$$
\left\|f_{n}-f_{\nu_{1}}\right\|<2^{-1}
$$

and then choose $\nu_{2}>\nu_{1}$ so that for $n>\nu_{2}$ we have

$$
\left\|f_{n}-f_{\nu_{2}}\right\|<2^{-2}
$$

and so on. Thus we may find a strictly increasing subsequence $\left\{\nu_{k}\right\}$ such that for $n>\nu_{k}$ we have

$$
\left\|f_{n}-f_{\nu_{k}}\right\|<2^{-k}
$$

and in particular

$$
\left\|f_{\nu_{k+1}}-f_{\nu_{k}}\right\|<2^{-k}
$$

whence

$$
\sum_{k=1}^{\infty}\left\|f_{\nu_{k+1}}-f_{\nu_{k}}\right\| \leq 1
$$

We write $f_{\nu_{m}}$ as a telescoping series $(k<m)$

$$
f_{\nu_{m}}=f_{\nu_{k}}+\sum_{j=k}^{m-1}\left(f_{\nu_{j+1}}-f_{\nu_{j}}\right)
$$

and consider the majorant (with $l=1$ )

$$
g_{\nu_{m}}=\left|f_{\nu_{k}}\right|+\sum_{j=k}^{m-1}\left|f_{\nu_{j+1}}-f_{\nu_{j}}\right|\left(\geq\left|f_{\nu_{m}}\right|\right)
$$

which is a monotone increasing sequence $\in A^{2} \cap \mathbb{R}$. For by the triangular inequality and (3.30)

$$
\left\|g_{\nu_{m}}\right\| \leq\left\|f_{\nu_{k}}\right\|+\sum_{j=k}^{m-1}\left\|f_{\nu_{j+1}}-f_{\nu_{j}}\right\| \leq\left\|f_{\nu_{k}}\right\|+1
$$

a.e., and a fortiori, bounded a.e., so that the sequence $\left\{g_{\nu_{m}}\right\}$ is convergent to $g(z)$, say. Hence $f_{\nu_{m}}$ in (3.31) is absolutely convergent as $m \rightarrow \infty$ to $f_{\infty}(z)$ a. e. . By the Lebesgue bounded convergence theorem and (3.33), we have

$$
\begin{aligned}
\|g(z)\|^{2} & =\int \lim _{m \rightarrow \infty} g_{\nu_{m}}(z)^{2} \mathrm{~d} x=\lim _{m \rightarrow \infty} \int g_{\nu_{m}}(z)^{2} \mathrm{~d} x=\lim _{m \rightarrow \infty}\left\|g_{\nu_{m}}\right\|^{2} \\
& \leq\left(\left\|f_{\nu_{k}}\right\|+1\right)^{2} .
\end{aligned}
$$


Hence $g \in A^{2}$. Since $\left|f_{\infty}\right| \leq g, f_{\infty} \in A^{2}$. Now letting $m \rightarrow \infty$ in (3.31), we have

$$
f_{\infty}-f_{\nu_{k}}=\sum_{j=k}^{\infty}\left(f_{\nu_{j+1}}-f_{\nu_{j}}\right) .
$$

Hence

$$
\left\|f_{\infty}-f_{\nu_{k}}\right\| \leq \sum_{j=k}^{\infty}\left\|f_{\nu_{j+1}}-f_{\nu_{j}}\right\| \rightarrow 0
$$

as $k \rightarrow \infty$, whence

$$
\lim _{k \rightarrow \infty} f_{\nu_{k}}=f_{\infty}
$$

From this we may conclude that

$$
\limsup _{n \rightarrow \infty}\left\|f_{\infty}-f_{n}\right\| \leq \lim _{k \rightarrow \infty}\left\|f_{\infty}-f_{\nu_{k}}\right\|+\lim _{k \rightarrow \infty, n>\nu_{k}}\left\|f_{\nu_{k}}-f_{n}\right\| \rightarrow 0,
$$

i.e. the sequence $\left\{f_{n}\right\}$ is convergent. There exists a subsequence $\left\{f_{\nu_{k}}\right\}$ which converges to $f_{\infty}$ a.e. in $D$ and the limit function satisfies (3.26).

Now for any open disc $D_{r}:|w-z|<r$, choose $D_{r} \subset D$. Then for $0<r_{0}<r$, we apply Lemma 3.5 with $D_{r_{0}}$ to obtain

$$
\left|f_{\nu_{k}}-f_{\nu_{l}}\right|^{2} \leq \frac{1}{\pi r_{0}^{2}} \iint_{\overline{D_{r_{0}}}}\left|f_{\nu_{k}}-f_{\nu_{l}}\right|^{2} \mathrm{~d} x \mathrm{~d} y \leq \frac{1}{\pi r_{0}^{2}} \iint_{D}\left|f_{\nu_{k}}-f_{\nu_{l}}\right|^{2} \mathrm{~d} x \mathrm{~d} y \rightarrow 0
$$

as $k, l \rightarrow \infty$. Hence $\left\{f_{\nu_{k}}\right\}$ is uniformly convergent on $D$ in the wide sense, which implies that $f_{\infty}(z)$ is regular in $D$. Hence every Cauchy sequence in $A^{2}$ converges.

Remark 3.7. If we replace the norm \|\| by the 2-norm, then the above proof also proves that the space $L^{2}(a, b)$ of all square integrable functions is a Hilbert space.

Suppose $h \in A^{2}$ and $h_{n}(z)=h(r) z^{n}$. Then similarly as in the proof of Lemma 3.5, we have with $r=|z|, D_{1}:|z|<1$ the inner product of $h_{m}, h_{n}$,

$$
\left(h_{m}, h_{n}\right)=\iint_{\bar{D}_{1}} h(r) \bar{h}(r) z^{m} \bar{z}^{n} \mathrm{~d} x \mathrm{~d} y=\delta_{m n} W_{n}
$$

where

$$
W_{n}=2 \pi \int_{0}^{1}|h(r)|^{2} r^{2 n+1} \mathrm{~d} r(>0) .
$$

Hence letting

$$
\alpha_{n}=\alpha_{n}(x, y)=\frac{1}{\sqrt{W_{n}}} h(r) z^{n}
$$

$\left\{\alpha_{n}\right\}$ forms an ONS by (3.38). Choose

$$
h=h(r)=(-\log r)^{s-\frac{1}{2}} r^{-1}, \quad \sigma=\operatorname{Re} s>0 .
$$

Then

$$
W_{n}=2 \pi(2 n)^{-2 \sigma} \Gamma(2 \sigma) .
$$

Indeed, in

$$
W_{n}=2 \pi \int_{0}^{1}(-\log r)^{2 \sigma-1} r^{2 n-1} \mathrm{~d} r
$$


we make the change of variable $-\log r=\frac{1}{2 n} u$ to deduce that

$$
W_{n}=2 \pi(2 n)^{-2 \sigma} \int_{0}^{\infty} u^{2 \sigma-1} e^{-u} \mathrm{~d} u
$$

which leads to (3.42). Hence (3.40) under the special choice of (3.41) amounts to

$$
\alpha_{n}(z)=\alpha_{n}(x, y)=\frac{n^{\sigma}}{\sqrt{(2 \pi) 2^{-2 \sigma} \Gamma(2 \sigma)}} \frac{(-\log |z|)^{s-\frac{1}{2}}}{|z|} z^{n} .
$$

In (3.16) we choose

(i) $\omega(n)=n^{-\sigma}$ resp. (ii) $\omega(n)=n^{-\sigma-1}$.

Then we obtain

$$
f_{n}(z):=\overline{\omega(n)}^{-1} \sum_{k=1}^{\infty} \omega(k) \alpha_{k n}(z)=\left\{\begin{array}{l}
n^{\sigma} \sum_{k=1}^{\infty} \frac{1}{k^{\sigma}} \alpha_{k n}(z) \\
n^{\sigma+1} \sum_{k=1}^{\infty} \frac{1}{k^{\sigma+1}} \alpha_{k n}(z)
\end{array}\right.
$$

Hence using $\alpha_{k n}=\frac{n^{\sigma}}{\sqrt{(2 \pi) 2^{-2 \sigma} \Gamma(2 \sigma)}} \frac{(-\log |z|)^{s-\frac{1}{2}}}{|z|} k^{\sigma} z^{k n}$, we have for $|z|<1$

$$
f_{n}(z)=\left\{\begin{array}{l}
\frac{n^{2 \sigma}}{\sqrt{(2 \pi) 2^{-2 \sigma} \Gamma(2 \sigma)}} \frac{(-\log |z|)^{s-\frac{1}{2}}}{|z|} \frac{z^{n}}{1-z^{n}} \\
-\frac{n^{2 \sigma+1}}{\sqrt{(2 \pi) 2^{-2 \sigma} \Gamma(2 \sigma)}} \frac{(-\log |z|)^{s-\frac{1}{2}}}{|z|} \log \left(1-z^{n}\right)
\end{array}\right.
$$

on finding the resulting sum $\sum_{k=1}^{\infty} z^{k n}$ resp. $\sum_{k=1}^{\infty} \frac{z^{k n}}{k}$.

Hence by Theorem 3.1, $\left\{f_{n}\right\}$ has the $D_{g}$ property, whence by Theorem 3.4, the sequence $\left\{\psi_{n}\right\}$ forms an ONS defined by

$$
\begin{aligned}
\psi_{n}(z)= & \frac{1}{\sqrt{(2 \pi) 2^{-2 \sigma} \Gamma(2 \sigma) \zeta(2 \sigma) \varphi_{2 \sigma}(n)}} \frac{(-\log |z|)^{s-\frac{1}{2}}}{|z|} \sum_{d \mid n} \mu\left(\frac{n}{d}\right) \frac{d^{2 \sigma} z^{d}}{1-z^{d}} \\
\psi_{n}(z)= & -\frac{1}{\sqrt{(2 \pi) 2^{-2 \sigma} \Gamma(2 \sigma) \zeta(2 \sigma+2) \varphi_{2 \sigma+2}(n)}} \frac{(-\log |z|)^{s-\frac{1}{2}}}{|z|} \\
& \left.\cdot \sum_{d \mid n} \mu\left(\frac{n}{d}\right) d^{2 \sigma+1} \log \left(1-z^{d}\right) \quad \text { ii }\right) .
\end{aligned}
$$

Here the $w$ in (3.15) is $w=\zeta(2 \sigma)$ or $w=\zeta(2 \sigma+2)$ and invoking (3.21), we have

$$
\varphi_{a}(n)=\Omega(n)=\sum_{d \mid n} \mu\left(\frac{n}{d}\right) d^{a}
$$

the Jordan totient function, where $a=2 \sigma$ or $a=2 \sigma+1$ according to the choice of $\omega(n)$ above.

Theorem 3.8. Let $\psi_{n}$ be defined by (3.48). Then $\left\{1, \psi_{n}(z), \psi_{n}(\bar{z})\right\}$ forms a complete ONS.

Completeness follows from the fact that the system $\left\{1, \frac{z^{n}}{1-z^{n}}, \frac{\bar{z}^{n}}{1-\bar{z}^{n}}\right\}$ forms a complete system since $\left\{1, z^{n}, \bar{z}^{n}\right\}$ does so. 
Now consider the boundary functions of the above $h_{n}=h_{n}(x)=e^{2 \pi i n x}, x \in(0,1)$, i.e. $L_{2}(0,1)$. The inner product (3.38) becomes

$$
\left(h_{m}, h_{n}\right)=\int_{0}^{1} e^{2 \pi i m x} e^{-2 \pi i n x} \mathrm{~d} x=\delta_{m n} W_{n} .
$$

The simplest ONS w.r.t. this inner product is $\alpha_{n}(x)=e^{2 \pi i n x}, W_{n}$ being $=1$. Hence with $\omega(n)=\frac{1}{n}$, we see that

$$
f_{n}(x)=n \sum_{k=1}^{\infty} \frac{e^{2 \pi i n k x}}{k}=n \log \left(1-e^{2 \pi i n x}\right), \quad x \notin \mathbb{Z}
$$

has the $D_{g}$-property with $g(n)=\zeta(2) n^{2}$ on noting that $w=\zeta(2)=\frac{\pi^{2}}{6}$. Hence we infer that

$$
\psi_{n}(x)=\frac{1}{\sqrt{\zeta(2) \varphi_{2}(n)}} \sum_{d \mid n} \mu\left(\frac{n}{d}\right) d \log \left(1-e^{2 \pi i d x}\right), \quad x \notin \mathbb{Z}
$$

forms an ONS. It is often more convenient to allow $z$ to take other complex values as long as the logarithm remains meaningful.

Note that if we choose $L^{2}(0,2 \pi)$, then $W_{n}=\frac{1}{\sqrt{2 \pi}}$ and $f_{n}$ and $\psi_{n}$ must be multiplied by $\frac{1}{\sqrt{2 \pi}}$ and so (3.52) should read

$$
\psi_{n}(x)=\frac{\sqrt{3}}{\pi^{\frac{3}{2}} \sqrt{\varphi_{2}(n)}} \sum_{d \mid n} \mu\left(\frac{n}{d}\right) d \log \left(1-e^{i d x}\right), \quad x \notin 2 \pi \mathbb{Z}
$$

as in [Ro51b, p. 144].

Example 3.9. Let

$$
Q_{n, k}(z)=\prod_{d \mid n}\left(1-z^{d}\right)^{\mu\left(\frac{n}{d}\right) d^{k}}, \quad Q_{n}(z)=Q_{n, 1}(z)=\prod_{d \mid n}\left(1-z^{d}\right)^{\mu\left(\frac{n}{d}\right) d} .
$$

The we have

$$
Q_{n}(z)=\prod_{d \mid n} \Phi_{d}(z)^{\varphi\left(\frac{n}{d}\right) d}
$$

where

$$
\Phi_{n}(z)=\prod_{d \mid n}\left(1-z^{d}\right)^{\mu\left(\frac{n}{d}\right)}
$$

is the nth cyclotomic polynomial, i.e. the polynomial containing all primitive nth roots of 1 and $\varphi(n)=\varphi_{1}(n)$, cf. (3.49), is the Euler function. Then (3.52) may be written as

$$
\psi_{n}(z)=\frac{1}{\sqrt{\zeta(2) \varphi_{2}(n)}} \sum_{d \mid n} \varphi\left(\frac{n}{d}\right) d \log \Phi_{d}(z)=\frac{1}{\sqrt{\zeta(2) \varphi_{2}(n)}} \log Q_{n}(z) .
$$

We may define the inner product so that $\left\{\log Q_{n, k}\right\}$ forms an ONS in $A^{2}(D)$.

Remark 3.10. Note that (3.47), (ii) resp. (3.51) $\left(-f_{1}(x)=\ell_{1}(x)\right)$ is essentially the polylogarithm function of order 1 and its boundary function. 


\section{Discrete Fourier transform}

The theory of discrete Fourier transform, DFT, is stated in many literature, [Fr99, pp.101-164], [Pa62], [W80, pp.89-109] and we will be brief. With the development of computers, fast computation (of DFT) is looked for and the Cooley-Tukey algorithm (Fast Fourier transform, FFT) and its generalizations have been prevailing [ATW82], [Bri74], [CT65], [DR90], [MS81], [Ros80], [SSW88], [W80]. For Fourier analysis on finite groups, cf. [C-SST08], [Ed72], [Her69], [Rud62], [Ter99] etc.

The theory of DFT, which is also known as the Finite Fourier Transform, for arithmetic functions has been developed in [LWK13] in the case of periodic functions and in [LLWK17, pp.109-114] in the case of a finite group. Cf. also [KaTs07, §8.1], [CKLW09, §§4.1, 4.3].

Definition 2. Let

$$
\varepsilon_{j}(x)=e^{2 \pi i j x / N}, \quad 1 \leq j \leq N,
$$

where $x$ is an integer variable and $N$ is a positive integer $\geq 2$. Then the set $\left\{\varepsilon_{j}(x) \mid 1 \leq j \leq N\right\}$ forms an orthogonal basis of the vector space $C(N)$ of all periodic arithmetic functions with period $N$, where an arithmetic function is one defined only for integer arguments. We define the discrete Fourier transform (DFT) $\hat{f}$ (or the yth Fourier coefficient) w.r.t. the $O N B\left\{\frac{1}{\sqrt{N}} \varepsilon_{j}(x) \mid 1 \leq j \leq N\right\}$ of $f$ by

$$
\hat{f}(y)=\frac{1}{\sqrt{N}} \sum_{x=0}^{N-1} \varepsilon_{y}(-x) f(x) .
$$

Then the Fourier inversion or Fourier expansion formula holds true:

$$
f(x)=\hat{\hat{f}}(-x)=\frac{1}{\sqrt{N}} \sum_{y=0}^{N-1} \varepsilon_{y}(x) \hat{f}(y) .
$$

DFT w.r.t. the orthogonal basis $\left\{\varepsilon_{j}(x) \mid 1 \leq j \leq N\right\}$ is also often used in much the same way as ordinary Fourier transforms.

One of the most prominent cases of applications of DFT to number theory is [Leh75] and subsequent [Fu90], both of which use (3.51) and have been incorporated as special cases of a more general theorem in [WAK20].

As Chen [Che10, pp.125-126] states, both arithmetical Fourier transform, AFT and FFT are algorithms for quick computation of DFT. We briefly state references on AFT, following [Che10]. Bruns [Br1903] proposed a method for calculating Fourier coefficients by Möbius inversion and Wintner [Wi47] re-established it for odd periodic function (without knowing the existence of Bruns' paper). Tufts and Sadasiv [TS88] repeated Wintner's argument with applications to VLSI. Schiff and Walker [SW92] extended Wintner's results to all periodic functions. After [RT90], it is termed AFT, cf. [Che10, pp. 125-147].

\section{References}

[Ah45] Ahiezer, N.I., General theory of Chebyshev polynomials, Scientific legacy of P.L. Chebyshev 1, Izd. Akad. Nauk SSSR, Moskwa-Leningrad, (1945) 5-42.

[Ah47] Ahiezer, N.I., Comments on the work of Chebyshev "On interpolation of quantities obtained by observations", Poln. Sobr. Soč. Chebysheva, Izd. Akad. Nauk SSSR, Moskwa-Leningrad, t. 2, (1947) 511.

[ATW82] Auslander, L., Tolimieri, R. and Winograd, S., . Hecke's theorem in quadratic reciprocity, finite nilpotent groups and the Cooley-Tukey algorithm, Adv. Math. 42, (1982) 123-172.

[Be76] Berndt, B.C., Classical theorems on quadratic residues, Enseign. Math. (2) 22, (1976), $261-304$.

[Bri74] Brigham, E.D., The fast Fourier transform, Prentice-Hall, New Jersey, 1974. 
[Br1903] Bruns, H. Grundlinien des wissenschaftlichen Rechnens, Teubner, Leipzig, 1903.

[CKLW09] Chakraborty, K., Kanemitsu, S, Li, J.-H. and Wang, X.-H., Manifestations of the Parseval identity, Proc. Japan Acad. Ser. A Math. Sci. 85 No. 9, (2009) 149-154.

[CKT09] Chakraborty, K., Kanemitsu, S. and Tsukada, H., Vistas of special functions II, World Sci., New Jersey-LondonSingapore etc., 2009.

[Ch20] Chan, H.H., Theta functions, elliptic functions and $\pi$, de Gruyter, Berlin/Boston, 2020.

[Chb1851] Chebyshev, P.L., Note sur le differentes séries, J. Math, Pures Appl. (1) 16, (1851) 337-346; Note on some series, Soč., t. I, StPb, (1899), 99-108; Poln. Sobr. Soč., Izd. Akad. Nauk SSSR, Moskwa-Leningrad t. 2 (1947), $244-313$.

[Chb1858] Chebyshev, P.L., On interpolation of quantities obtained by observations, Bull. Phys-Math. Akad. Nauk StPb 16 (1858), 353-358; Soč., t. I, StPb, (1899), 711-714; Poln. Sobr. Soč. Izd. Akad. Nauk SSSR, Moskwa-Leningrad t. 2 (1947), 239-243.

[Chb1859] Chebyshev, P.L., On interpolation in the case of a large number of data obtained by observations, Soč., t. I, StPb, (1859), 387-469; Poln. Sobr. Soč. Izd. Akad. Nauk SSSR, Moskwa-Leningrad t. 2 (1947), 244-313.

[Che10] Chen, N.-X., Möbius inversion in physics, World Sci., New Jersey-London-Singapore etc., 2010.

[CT65] Cooley, J.W. and Tukey, J.W., An algorithm for machine calculation of complex Fourier series, Math. Comp. 19, (1965), 297-301.

[DR90] Diaconis, P. and Rockmore, D., Efficient computation of the Fourier transform on finite groups, J. Amer. Math. Soc. 3, (1990), 297-332.

[Fr99] Frazier, M.W., An introduction to wavelettes through linear algebra, Springer, New York etc., 1999.

[Fu90] Funakura, T., On Kronecker's limit formula for Dirichlet series with periodic coefficients, Acta Arith. 55, (1990), 59-73.

[KaKi95] Kanemitsu, S. and Kiuchi, I., Annotated translation with addition of N. P. Romanoff: Hilbert space and number theory, Mem. Fac. Lib. Arts, Ser. Natur. Sci. 29, (1995), 13-54.

[KaTs07] Kanemitsu, S. and Tsukada, H., Vistas of special functions, World Sci., New Jersey-London-Singapore etc., 2007.

[KaTs14] Kanemitsu, S. and Tsukada, H., Contributions to the theory of zeta-functions: the modular relation supremacy, World Sci., Singapore etc., 2014.

[KiOn61] Kiselev, A.A. and Onufrieva, L.A., Applications of biorthogonal systems of Chebyshev and Markov for approximation of functions, In Studies on recent problems of constructive theory of functions, Fizmatgiz, Moskwa, (1961), 183-189.

[LaGa02] Laurinchikas, A. and Garunkstis, R., The Lerch zeta-function, Kluwer Academic Publ., Dordrecht-Boston-London, 2002 .

[Leh75] Lehmer, D.H., Euler constants for arithmetic progressions, Acta Arith. 27, (1975), 125-142; Selected Papers of D.H. Lehmer, Vol. II, 591-6oe, Charles Babbage Res. Center, Manitoba 1981.

[Lew80] Lewin, L., Structural properties of polylogarithms, AMS, Providence, 1980.

[LWK13] Li, F.-H., Wang, N.-L. and Kanemitsu, S., Number theory and its applications, World Sci., London-Singapore-New Jersey, 2013.

[LLWK17] Li, H.-L., Li, F.-H., Wang, N.-L. and Kanemitsu, S., Number theory and its applications II, World Sci., LondonSingapore-New Jersey, 2017.

[Li1909] Liebmann, E.D., Vereinfachte Behandlung einiger Minimalproblems von Tschebyscheff, Jahresber. Deutsch. Math. Verein. 18, (1909), 433-449.

[Ma1895] Markov, A.A. (senior), On limit values of integrals, Izv. Akad. Nauk StPb. Ser. V 2, (1895), 195-203; In Izbr. Trudy, Gostexizd, Moskwa-Leningrad, 146-230.

[Ma1898] Markov, A.A. (senior), On limit values of integrals in connection with interpolation, Zap. Akad. Nauk po. Fyzikomatemam. Otd. StPb. Ser. VIII 6, No. 5, (1898) 69 pp.; In Izbr. Trudy, Gostexizd, Moskwa-Leningrad, 146-230; Recherches sur les valuers extrème des intégrales et sur l'interpolation, Acta Math. 28 (1904), 243-301.

[MS81] Merseau, R.M. and Speake, T.C., A unified treatment of Cooley-Tukey algorithm for evaluation of the multidimensional DFT, IEEE Trans. Acoustics and Signal Processing29, No. 5, (1981), 1011-1017.

[M54] Müller, M., Über die Konvergenz eines Verfahrens zur Berechnung der Fourier-Koeffizient Math. Z. 60, (1954), 81-87.

[On83] Onufrieva, L.A., The Chebyshev interpolation method in the case of a large number of data, (Russian) Istor.-Mat. Issled. 27, (1983), 259-274.

[Pa62] Papoulis, A., The Fourier integral and its applications, McGraw-Hill, 1962.

[Rad1922] Rademacher, H., Einige Sätze über Reihen von allgemeinen Orthogonalfunktionen, Math. Ann. 87, (1922), 112-138.

[RT90] Reed, I.S., Tufts, D.W. et al, Fourier analysis and signal processing by use of Möbius inversion formula, IEEE Trans. Acoustics, Speech, 6 Signal Processing 38, (1990), 458-470.

[Ro51a] Romanoff, N.P., Hilbert spaces and number theory I, Izv. Akad. Nauk SSSR, Ser. Mat. 10 (1951), 3-34. 
[Ro51b] Romanoff, N.P., Hilbert spaces and number theory II, Izv. Akad. Nauk SSSR, Ser. Mat., 15, (1951), 131-152.

[Ros80] Rose, D.J., Matrix identities of the fast Fourier transform, Lin. Alg. Appl. 29, (1980), 423-443.

[SSW88] Schiff, J.L., Surendok, T.J. and Walker, R.W., An algorithm for computing the inverse Z-transform, IEEE Trans. Signal Processing 40, (1988), 2194-2198.

[SW92] Schiff, J.L. and Walker, R.W., A sampling theorem and Wintner's results on Fourier coefficients, J. Math. Anal. Appl. 133, (1992), 466-471.

[TS88] Tufts, D.W. and Sadasiv, G., The arithmetic Fourier transform, IEEE Trans. Acoustics, Speech, \& Signal Processing Magazine 5, (1988), 13-17.

[WAK20] Wang, N.L., Agarwal, P. and Kanemitsu, S., Limiting values and functional and difference equations, Mathematics, Difference and Differential Equations, 8, (2020), 407; doi:10.3390/math8030407.

[WMK20] Wang, X.-H, Mehta, J. and Kanemitsu, S., The boundary Lerch zeta-function and short character sums à la Y. Yamamoto, Kyushu J. Math. 74 (2020), 313=-335, doi:10.2206/kyushujm.74.313.

[W80] Winograd, S., Arithmetic complexity of computations, SIAM, Philadelphia, 1980.

[Wi41] Wintner, A., On Riemann's fragment concerning elliptic modular functions, Amer. J. Math. 63, (1941), 628-634.

[Wi44] Wintner, A., Diophantine approximations and Hilbert's space, Amer. J. Math. (3) 66, (1944), 564-578.

[Wi47] Wintner, A., An arithmetical approach to ordinary Fourier series, Wavery Press, Baltimore, 1947.

[Ya77] Yamamoto Y., Dirichlet series with periodic coefficients, Proc. Intern. Sympos. "Algebraic Number Theory", Kyoto 1976, 275-289. JSPS, Tokyo., 1977.

\section{Harmonic analysis on groups}

[C-SST08] Ceccherini-Silberstein, T., Scarabotti, F. and Tolli, F. Harmonic analysis on finite groups, Cambridge UP, Cambridge etc., 2008 .

[Ed72] Edwards, R.E., Integration and harmonic analysis on compact groups, Cambridge UP, Cambridge etc., 1972.

[Her69] Hermann, R., Fourier analysis on groups and partial wave analysis, Benjamin, New York, 1969.

[Rud62] Rudin, W. Fourier analysis on groups, Wiley, New York, 1962.

[Ter99] Terras, A., Fourier analysis on finite groups and applications, Cambridge UP, Cambridge etc., 1999.

\section{J.-W. Feng}

University of Science and Technology of China

96 Jinzhai Road, Hefei

Peoples Republic of China

e-mail: 291569346@qq.com

\section{S. Kanemitsu}

Faculty of Engineering

Kyushu Institute of Technology

Kitakyushu 804-0855, JAPAN

e-mail: omnikanemitsu@yahoo.com

\section{T. Kuzumaki}

Applied Physics Course

Faculty of Engineering, Gifu University,

Gifu 500-1193, JAPAN

e-mail: kuzumaki@gifu-u.ac.jp 